\title{
Outcomes of Bridging Roles in MNCs and Moderating Influence of Cultural Identity Integration
}

$\operatorname{AUTHOR}(\mathrm{S})$ :

Liu, Ting; Sekiguchi, Tomoki; Ebisuya, Azusa

\section{CITATION:}

Liu, Ting ... [et al]. Outcomes of Bridging Roles in MNCs and Moderating Influence of

Cultural Identity Integration. Academy of Management Proceedings 2019, 2019(1): 18080.

\section{ISSUE DATE:}

2019-08

URL:

http://hdl.handle.net/2433/252531

\section{RIGHT:}

Copyright of Academy of Management Journal is the property of Academy of Management and its content may not be copied or emailed to multiple sites or posted to a listserv without the copyright holder's express written permission. However, users may print, download, or email articles for individual use.; The full-text file will be made open to the public on 1 August 2020 in accordance with publisher's 'Terms and Conditions for Self-Archiving'.; This is not the published version. Please cite only the published version.; この論文は出版社版でありません。引用の際には出版社版 をご確認ご利用ください。 


\title{
OUTCOMES OF BRIDGING ROLES IN MNCS AND MODERATING INFLUENCE OF CULTURAL IDENTITY INTEGRATION
}

\author{
TING LIU \\ Department of International Studies, Hiroshima City University, \\ 3-4-1, Ozuka-Higashi, Asa-Minami-Ku, Hiroshima, 731-3194, JAPAN \\ TOMOKI SEKIGUCHI \\ Kyoto University \\ AZUSA EBISUYA \\ Osaka University
}

INTRODUCTION

Today, multinational companies (MNCs) increasingly recognize the important role of individuals who internalize and identify with multiple cultures and languages (Brannen \& Thomas, 2010; Fitzsimmons, Liao, \& Thomas, 2017). As expatriates, inpatriates, or nonnative locals, these individuals often become bridge individuals, or individuals who have specific cultural and language skills and who act in bridging roles between different cultural and/or language groups in MNCs (Harzing, Köster, \& Magner, 2011, Sekiguchi, 2016). Because of their multidimensional activities, bridge individuals are seen as knowledge-transferring nodes, gatekeepers, conduits for resource acquisition, and influence agents (Aldrich \& Herker, 1977; Ancona \& Caldwell, 1992).

Because the concept of bridging roles and bridge individuals in the international business context has not received much attention from international business researchers until now with a few exceptions (e.g., Harzing et al., 2011; Sekiguchi, 2016), the concepts themselves and their roles are insufficiently investigated. Therefore, we do not have a clear understanding of the concept of the bridging role including its underlying dimensions and the possible consequences of performing a bridging role by bridge individuals. To address this research gap, we employ a mixed method approach by conducting both qualitative and quantitative investigations to generate and test a new theory regarding the effect of the bridging role performed by bridge individuals.

We first conducted a preliminary qualitative study with 60 bridge individuals working in 13 Japanese foreign subsidiaries because the lack of extant literature on this topic prevented us from deductively developing a theory and hypotheses. The findings from the preliminary study contributed to the refinement of the concept of the bridging roles, the understanding of the potential positive and negative consequences brought to the bridge individuals when they perform the bridging roles, and the importance of cultural identity held by the bridge individuals. These findings also enabled us to develop a new theory and hypotheses by integrating the insights with the existing theoretical frameworks, namely, social network theory, job demands-resources (JD-R) theory, and the perspective of cultural identity integration. We then tested our predictions quantitatively using a sample of 200 Japanese employees working in Japanese overseas subsidiaries and the results are generally supportive.

Using both qualitative and quantitative methodologies, the present study contributes to the international business literature for several ways. First, our study explores the under-investigated concept of the bridging role and bridge individuals in the context of MNCs and provides novel conceptualizations of the bridging role. Second, we develop and empirically demonstrate a new theoretical framework concerning both the positive and negative consequences of performing 
functional and linguistic bridging by bridge individuals. Third, we incorporate the concept of cultural identity into our theoretical framework and demonstrate its moderating influence on the effects of the bridging role. In short, our study adds significant value to the deeper understanding of the bridging role and bridge individuals in MNCs.

\section{STUDY1: PRELIMINARY QUALITATIVE STUDY}

Utilizing the grounded theory approach (Glaser \& Strauss, 1967; Strauss \& Corbin, 1998), we conducted a preliminary qualitative study without any specific theory or hypotheses in mind. The study was guided by the following research question: What is the essential nature of bridging activities in MNCs and what are the potential consequences when individuals perform bridging roles?

\section{Data Collection and Data Analysis}

We collected data with semi-structured interviews with 60 employees in 13 Japanese subsidiaries in Germany and the Netherlands. All of our interviewees can be considered bridge individuals because they were playing a role of improving the company and individual performance through bridging roles.

Data analysis was conducted in an iterative process. We used the Atlas.ti qualitative research software to analyze our interview data. First, we used the open coding technique to categorize the interview data (Charmaz, 2010; Locke, 2001; Strauss \& Corbin, 1998), and we created first-order coding for each concept. Then we merged our first-order concepts to reduce their number by comparing the similarities and differences. Based on the first-order coding, we defined second-order focus points. Finally, we summarized the second-order concepts into our core focus.

\section{Summary of Findings and Insights}

We found that the bridge individuals in our target MNCs play functional and linguistic bridging roles, and these activities have both positive (i.e., high quality of TMX) and negative consequences (i.e., emotional exhaustion due to the role conflict) for the individuals. We also obtained some insights into the fact that the cultural identity of bridge individuals may influence the effect of bridging roles on the individual outcomes. In the main study described below, we integrate these preliminary findings with the existing theories to build a theoretical model and test the hypotheses using quantitative data.

\section{THEORY ELABORATION AND HYPOTHESES}

By elaborating upon the findings from the preliminary qualitative study, we develop and test the theoretical framework concerning the potential positive and negative consequences of the performance of bridging roles by bridge individuals.

\section{The Positive Effect of Bridging Roles}

Drawing on the social network theory, we theorize that performing functional and linguistic roles by bridge individuals can promote TMX through the development of social capital at the workplace. According to social network theory, there are many types of clusters, which can be 
different departments or ethnic groups in an organization (Cross, Parker, \& Sasson, 2003). Social network theory explains that bridge individuals act as a connection between clusters within the organization (Sekiguchi, 2016). In MNCs, clusters include not only the departments, divisions, and teams, but also different cultural and language groups. From the preliminary study, both the functional bridging and linguistic bridging individuals can be seen as ties, connecting clusters in MNCs. These two types of bridge functions can facilitate information spreading and diffuse norms and values.

Lin (1999) defined social capital as access to and use of resources embedded in social networks. Investment in social networks can facilitate the flow of information and provide useful information regarding opportunities. Bridge individuals can be seen as connectivity in a network, carrying information, resources, etc. through the network (Moody \& Paxton, 2009). Thus, bridge individuals' social tie resources and their acknowledged relationships can be conceived as certifications of social credentials by the organization or its members. Therefore, bridge individuals have more accessibility to resources through social networks reflecting their social capital (Lin, 1999).

TMX represents an individual's perception of the exchange quality of his or her role in relationship interactions with other team members (Seers, 1989). Individuals who invest more effort into valuing high-quality relationships within the team will receive respect from team members, and this will encourage some individuals to forge strong and harmonious relationships for TMX (Banks et al., 2014). As we argued in the preliminary study, bridge individuals are ties and bridges in organizations, and they facilitate social attachment and contribute more in working relationships. They establish high-quality interactions within intergroup relations with team members via the resources they are embedded in, which can be seen as social capital. Thus, we hypothesize:

Hla: Functional bridging is positively related to TMX, mediated by social capital at the workplace.

H1b: Linguistic bridging is positively related to TMX, mediated by social capital at the workplace.

\section{The Dark Side of Bridging Roles}

Drawing on job demands-resources (JD-R) theory (Bakker \& Demerouti, 2007; Bakker, Demerouti, De Boer, \& Schaufeli, 2003; Bakker, Demerouti, \& Verbeke, 2004) we predict that performing functional and linguistic bridging by bridging individuals causes emotional exhaustion among bridge individuals through the increasing level of role conflict.

JD-R theory states that stressors in the workplace are produced by the relationship between two categories: job demands and job resources. Based on JD-R theory, job demands include work overload, role conflict, and emotional demands that require effort and skills from bridge individuals. Job resources, on the other hand, represent all aspects of organizational characteristics that can facilitate the completion of the job and reduce emotional demands. As we found in the preliminary study, when bridge individuals act as bridges among clusters in the organization, not only do positive effects occur, but negative influence also occurs. Because bridge individuals perform their bridging role as part of their normal job (Harzing et al., 2011), when their capacity cannot meet the requirements from both their main job and their bridging job, the bridging role will cause job stressors and role conflict will happen. When an inadequate dyadic relationship happens 
between the bridging role and the resources they have, role conflicts occur, as job demands can further intensify emotional exhaustion (Maslach, Schaufeli, \& Leiter, 2001). Thus, we hypothesize,

H2a: Functional bridging is positively related to emotional exhaustion, mediated by role conflict.

H2b: Linguistic bridging is positively related to emotional exhaustion, mediated by role conflict.

\section{Moderating Role of Cultural Identity Integration}

Networks are increasingly seen as a structure of clusters of diverse individuals, groups, and organizations working collaboratively. Bridge individuals occupy key structural positions in networks, affording opportunities and constraints on their actions. The social network approach advocates for individuals to be identified and provides information and materials on processing communication and interventions to amplify networking functions (Long, Cunningham, \& Braithwaite, 2013).

Mok and colleges (2007) demonstrated that bicultural identity integration influences whom bicultural people interact with. Their research indicates that bicultural people with more integrated cultural identity interact with more host culture members to verify their embracing of the host country culture. Regarding the bridging roles in MNCs, a highly integrated cultural identity will diminish the bridging role because bridging roles only focus on the mixing and blending of interactions rather than the dyadic bridging of both home and host clusters. In this situation, both the home country employees and host country employees will view bridge individuals as outsiders.

Adaptability and linguistic bridging are highest for individuals with the most inconsistent cultural identity integration (Fitzsimmons et al., 2017). This means employees who have a low cultural identity integration can straddle two cultures and switch the role being played as needed; therefore, low cultural identity integration helps amplify the effect of social capital on TMX.

Emotional exhaustion is generally observed due to higher job demands and insufficient job resources available to employees (Hu, Schaufeli, \& Taris, 2011). Cultural and language skills also serve as key factors and resources in reducing emotional exhaustion. Low cultural identity as a cultural skill can diminish the influence role conflicts have on emotional exhaustion. Thus, we hypothesize the moderating role of cultural identity integration as,

H3a: The indirect effect of functional bridging on TMX, via social capital, is moderated by cultural identity integration such that the indirect effect is stronger when cultural identity integration is low, but weakens when cultural identity integration is high.

H3b: The indirect effect of linguistic bridging on TMX, via social capital, is moderated by cultural identity integration such that the indirect effect is stronger when cultural identity integration is low, but weakens when cultural identity integration is high.

H4a: The indirect effect of functional bridging on emotional exhaustion, via role conflict, is moderated by cultural identity integration such that the indirect effect is stronger when cultural identity integration is high, but weakens when cultural identity integration is low. 
H4b: The indirect effect of linguistic bridging on emotional exhaustion, via role conflict, is moderated by cultural identity integration such that the indirect effect is stronger when cultural identity integration is high, but weakens when cultural identity integration is low.

\section{STUDY2: HYPOTHESES TESTING WITH QUANTITATIVE DATA}

\section{Participants and Procedure}

All the respondents for Study 2 were recruited through a market research company based in Japan. Since most of the respondents in this company are Japanese, we narrowed our research subject to Japanese employees working in the Japanese foreign subsidiaries. We received responses from 200 Japanese employees working in overseas Japanese subsidiaries.

\section{Measures and Results}

All measures (functional bridging, linguistic bridging, social capital, role conflict, cultural identity integration, TMX, emotional exhaustion, and control variables) except for control variables and linguistic bridging were measured using a seven-point Likert scale ranging from 1 (strongly disagree) to 7 (strongly agree).

Prior to hypothesis testing, we first conducted a confirmatory factor analysis using the lavaan package in $\mathrm{R}$ (Rosseel, 2012) to ensure that the seven variables in the model had adequate validity. To test Hypotheses 1a, 1b, 2a, and 2b, we conducted bootstrapping-based mediation tests using the PROCESS macro (Hayes, 2013). To test Hypotheses 3a, 3b, 4a and 4b, we then utilized the method introduced by Hayes (2013) to test the conditional indirect effects of functional and linguistic bridging on TMX and emotional exhaustion through their mediators at one standard deviation above and below the mean of the moderator terms with bootstrapping-based moderated mediation tests using the PROCESS macro (Hayes, 2013). In summary, all the hypotheses except Hypothesis $4 \mathrm{a}$ were supported by the current data analysis.

\section{DISCUSSION}

\section{Theoretical Contributions}

The current study contributes to the literature on bridge individuals and their bridging roles in the international business context. First, after the closer look at what bridge individuals actually do in MNCs through the qualitative investigation, we could obtain new insights to the existing concepts of bridge individuals and their bridging roles (Harzing, Köster, \& Magner, 2011, Sekiguchi, 2016). Our new conceptualizations of bridging roles performed by bridge individuals involve two functional bridging and linguistic bridging. Whereas the functional bridging is the similar activities with boundary spanning (Ancona \& Caldwell, 1992), linguistic bridging is more akin to what Harzing and her colleagues (2011) refer the activities that bridge individuals perform. These conceptual differences within the domain of bridging roles in MNCs would have significant theoretical implications especially for future research.

Second, based on the preliminary findings from the qualitative study about what are brought to bridge individuals when they perform functional and linguistic bridging roles, we could generate and elaborate on the theoretical framework regarding the effects of bridging roles on bridge individuals' interpersonal and emotional outcomes. Especially, our new theoretical 
perspective suggests that for bridge individuals, performing bridging roles does not always produce desirable outcomes for them. Our findings shed light on the complex nature of bridging roles in MNCs.

Furthermore, we delineated and empirically demonstrated the moderating role of cultural identity integration within bridge individuals on the relationship between bridging roles and subsequent individual outcomes. Our finding is consistent with Fitzsimmons and colleges (2017)'s articulation that individuals who have a separate cultural identity are more likely to associate with different cultures and bridge networks. Related to the view that cultural skills can be the resources in promoting boundary spanning activities, our research suggests that, when the cultural identity integration is low, bridge individuals are able to switch their different cultural identity according to the groups they communicate, which enables them to bridge different cultural and possibly linguistic groups.

Finally, language research is becoming incrementally prominent in international business studies (Tenzer, Terjesen, \& Harzing, 2017). In this regard, our conceptualization of the linguistic bridging role as a new perspective would contribute to the clarification of the bridging constructs guiding future research such that the linguistic bridging role is more related to the language issues than the functional bridging role. In addition, given the close relationship between language and culture, our conceptualization of linguistic bridging would stimulate new research on multiculturalism and multilingualism, as we theorized and tested the model involving linguistic bridging and cultural identity identification.

\section{Practical Implications}

Our findings also have practical implications both from the employee and MNC perspectives. First, from the employee perspective, our findings suggest that performing bridging roles in MNCs such as in headquarters or foreign subsidiaries have a profound impact on their social capital, which not only helps MNCs to manage international operation smoothly but also is pivotal for their own career advancement within MNCs. However, employees who are willing to serve as bridge individuals should also acknowledge the potential dark side of the bridging roles, such as the high risk of having emotional exhaustion and burnout as the findings of our study indicate. In addition, our conceptualizations of bridging roles indicate that what kinds of skills and abilities would be the valuable resources to be the effective bridge individuals. Even without such attributes, certain employees could become bridge individuals if they are skillful in the functional bridging role.

Second, from the MNC perspective, we contend that MNCs can benefit from selecting and developing bridge individuals carefully by keeping our findings in mind. For example, our study demonstrated that having competent bridge individuals will promote the good interpersonal relationships within and between different groups within MNCs. Especially noteworthy is our finding on the moderating effect of cultural identity integration. That is, our findings indicate not all types of multicultural employees can be effective bridge individuals. Rather, employees with the low level of cultural identity integration will do the bridging roles more effectively because they are more likely than those who are high in cultural identity integration to develop better social capital and TMX, and less likely to experience emotional exhaustion caused by the perception of role conflict.

\section{REFERENCES AVAILABLE FROM THE AUTHORS}

